\title{
Turbelários límnicos (Platyhelminthes) em ecossistemas de arroz irrigado da Planície Costeira do sul do Brasil
}

\author{
Dioneia Conceição da Vara ${ }^{1}$ \& Ana Maria Leal-Zanchet ${ }^{1,2}$

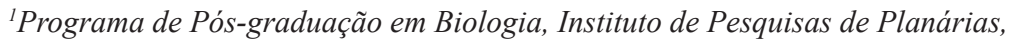 \\ Universidade do Vale do Rio dos Sinos - UNISINOS, CEP 93022-000, \\ São Leopoldo, RS, Brasil. http://www.unisinos.br \\ ${ }_{2}^{2}$ Autor para correspondência: Ana MariaLeal-Zanchet,e-mail: zanchet@unisinos.br
}

VARA, D.C. \& LEAL-ZANCHET, A.M. Freshwater turbellarians (Platyhelminthes) in irrigated rice fields of the Coastal Plain in southern Brazil. Biota Neotrop. (13)4: http://www.biotaneotropica.org.br/v13n4/en/ab stract?inventory+bn02013042013

\begin{abstract}
Turbellarians occur in a variety of wetlands, being also abundant in temporary water bodies. Several turbellarians are top predators in their microhabitats, many of them feeding on zooplankton. However, they are seldom taken into account in biodiversity studies. Information on turbellarian diversity in Brazilian freshwater ecosystems mainly came from taxonomical studies. In southern Brazil, there are a high number of wetlands, most of them represented by rice fields. Despite their impact on natural areas, these managed ecosystems can play an important role in freshwater biodiversity conservation. The aim of the present work was to report on an inventory done in irrigated rice fields of three areas of the Coastal Plain of southern Brazil, viz. Cachoeirinha, Santo Antônio da Patrulha e Camaquã. We found 144 species of freshwater turbellarians, distributed in six taxonomic groups (Catenulida, Macrostomida, Lecithoepitheliata, Proseriata, Rhabdocoela, and Tricladida). Twenty-three species are recorded for the first time for Brazil. Catenulida showed the highest relative abundance in Cachoeirinha, followed by Rhabdocoela. In Santo Antônio and Camaquã, Tricladida had the highest relative abundance, followed by Catenulida. Results suggest that some turbellarian species show a high level of habitat specificity with only $38 \%$ of the registered turbellarians occurring in a single study area. Although there is a paucity of turbellarian inventories in Brazilian natural wetlands, hampering a comparison between natural and managed wetlands, results indicate high species richness in the areas studied.
\end{abstract}

Keywords: microturbellarians, triclads, diversity, inventory, temporary wetlands.

VARA, D.C. \& LEAL-ZANCHET, A.M. Turbelários límnicos (Platyhelminthes) em ecossistemas de arroz irrigado da Planície Costeira do sul do Brasil. Biota Neotrop. 13(4): http://www.biotaneotropica.org.br/v13n4/ pt/abstract?inventory+bn02013042013

Resumo: Os turbelários são encontrados em uma grande variedade de hábitats aquáticos, sendo abundantes inclusive em áreas úmidas temporárias. Diversos turbelários são predadores de topo nos seus microhabitats, muitos deles alimentando-se de zooplâncton. No entanto, raramente os turbelários são considerados em inventários de diversidade. Informações sobre sua diversidade nos ecossistemas límnicos brasileiros provêm principalmente de estudos taxonômicos. No Rio Grande do Sul, há um grande número de áreas úmidas, sendo a maioria delas representadas por lavouras de arroz. Esses ecossistemas manejados, embora tenham alto impacto sobre áreas naturais, podem exercer um importante papel na conservação da biodiversidade aquática. Este estudo teve por objetivo inventariar os turbelários ocorrentes em áreas de cultivo de arroz irrigado de três localidades da Planície Costeira do sul do Brasil (Cachoeirinha, Santo Antônio da Patrulha e Camaquã). Foram registradas 144 espécies de turbelários, distribuídas em seis grupos taxonômicos (Catenulida, Macrostomida, Lecithoepitheliata, Proseriata, Rhabdocoela e Tricladida). Vinte e três espécies são registradas pela primeira vez para o Rio Grande do Sul. Catenulida apresentou a maior abundância relativa em Cachoeirinha, seguida por Rhabdocoela, enquanto nas outras duas localidades, a maior abundância relativa foi de Tricladida, seguida por Catenulida. Os resultados sugerem que algumas espécies demonstram alto grau de especificidade de hábitat, pois cerca de $38 \%$ dos turbelários ocorreram em apenas uma das áreas de estudo. Apesar da existência de poucos inventários de turbelários em áreas úmidas naturais do Brasil, impedindo uma comparação entre áreas úmidas naturais e manejadas, os resultados indicam uma elevada riqueza de espécies de turbelários nas áreas de estudo.

Palavras-chave: microturbelários, tricladidos, diversidade, inventário, áreas úmidas temporárias. 


\section{Introdução}

No Brasil, estudos taxonômicos sobre turbelários límnicos foram realizados principalmente nos estados de São Paulo (Marcus 1943, 1944, 1945a, b, 1946, 1948, 1949, 1950, 1952, 1954, DuBois Reymond Marcus \& Marcus 1951) e Rio Grande do Sul (Kawakatsu 1989, Kawakatsu et al. 1976, 1980, 1982, 1983a, b, 1984, 1985, 1986, 1992, Sluys et al. 1997, Gamo \& Leal-Zanchet 2004, Amato \& Amato 2005, Amato et al. 2003, 2005, 2006, 2007, 2010, 2011, Seixas et al. 2010a, b, c, 2011). Há registros de 98 espécies de turbelários dulcícolas para o Brasil, sendo os macroturbelários representados por 12 espécies e os microturbelários por 86 espécies formalmente descritas (Carbayo et al. 2009). Em ambientes límnicos, os macroturbelários incluem representantes de Tricladida e os microturbelários, de Catenulida, Macrostomida, Prolecithophora, Lecithoepitheliata, Proseriata e Rhabdocela (Young 2001). Segundo Noreña et al. (2003), das 510 espécies de microturbelários citadas para as regiões do Gondwana, mais de $45 \%$ foram registradas no Brasil.

No entanto, mesmo nas regiões com maior número de registros de turbelários límnicos no país, o conhecimento da sua diversidade baseia-se principalmente em dados coletados a partir de amostragens pontuais, havendo escassos inventários até o momento. Para os trabalhos taxonômicos sobre tricladidos límnicos, coletas diretas foram realizadas em ambientes lóticos e lênticos, especialmente na cidade de São Paulo (Marcus 1946) e nas sub-bacias dos rios Caí e Sinos, nordeste do Rio Grande do Sul (Kawakatsu et al. 1980, Sluys et al. 1997). Estudos taxonômicos realizados sobre microturbelários em geral, mais extensivos em São Paulo, foram desenvolvidos com base em coletas em ambientes lóticos nos arredores da cidade de São Paulo (Marcus 1943, 1944, 1945a, b, 1946, 1948, 1949, 1950, 1952, 1954; Du-Bois Reymond Marcus \& Marcus 1951). Os trabalhos taxonômicos sobre temnocefalídeos foram realizados com base na coleta de seus hospedeiros, em especial no Rio Grande do Sul (Amato \& Amato 2005, Amato et al. 2003, 2005, 2006, 2007, 2010, 2011, Seixas et al. 2010a, b, c, 2011). Adicionalmente, Gamo \& Leal-Zanchet (2004) realizaram um rápido inventário de microturbelários em sete localidades do Rio Grande do Sul e Braccini \& Leal-Zanchet (2013) estudaram a estrutura de comunidades de turbelários em nove lagoas na Planície Costeira desse estado.

Os turbelários representam um importante componente da comunidade bentônica nos ecossistemas límnicos. Sua ocorrência tem sido registrada em ambientes lóticos e lênticos, em ecossistemas naturais permanentes e temporários (Kolasa 1991, Noreña-Janssen 1995, Young 2001) e em agroecossistemas (Bambaradeniya et al. 2004). Os microturbelários são capazes de colonizar e sobreviver em ambientes temporários, devido apresentarem boa capacidade de dispersão, ciclo de vida versátil, formação de ovos resistentes à dessecação e altas taxas de reprodução e desenvolvimento (Young 2001). Já os macroturbelários apresentam baixa capacidade de dispersão e, embora não tenham formas de resistência à dessecação, podem apresentar reprodução assexuada (Ball \& Reynoldson 1981), o que facilita a colonização de determinados ambientes.

Os microturbelários alimentam-se de algas, bactérias, protozoários, nematódeos, oligoquetos, microcrustáceos, larvas de insetos, outros microturbelários, além de algas e/ou bactérias, dentre outros organismos (Young 2001), enquanto os macroturbelários alimentam-se principalmente de outros invertebrados bentônicos (Ball \& Reynoldson 1981). Assim, estudos da biodiversidade de turbelários podem indicar a biodiversidade de outros grupos e auxiliar no monitoramento de comunidades. No entanto, apesar de sua importância ecológica, abundância e riqueza de espécies em ecossistemas límnicos, os tubelários raramente são considerados em inventários de diversidade (Schockaert et al. 2008).

No Rio Grande do Sul, há um grande número de áreas úmidas (Diegues 1990, Maltchik et al. 2003), naturais e manejadas, com uma superfície aproximada de $30.300 \mathrm{~km}^{2}$, distribuídas principalmente na Planície Costeira, Depressão Central e Fronteira Oeste (Maltchik et al. 2003). Das cinco classes principais de áreas úmidas identificadas, os arrozais perfazem aproximadamente $84 \%$ da área de inundação do estado (Maltchik et al. 2003). A orizicultura irrigada pode desempenhar um importante papel na conservação da biodiversidade (Maeda 2001, Tourenq et al. 2001, Czech \& Parsons 2002, Elphick \& Oring 2003, Guadagnin \& Maltchik 2007), mas, de fato, pouco é conhecido sobre esse tema em arrozais do sul do Brasil. De modo geral, dados sobre a diversidade associada às lavouras de arroz são escassos (Elphick 2000, Blanco et al. 2006). A maioria dos inventários de invertebrados no cultivo do arroz irrigado relaciona-se a organismos que causam prejuízos econômicos (Bambaradeniya et al. 2004) ou danos à saúde humana (Forattini et al. 1989).

Considerando que os arrozais ocupam a maior parte da área de inundação do Rio Grande do Sul, inventariamos, no presente estudo, os turbelários ocorrentes em lavouras de arroz e seus canais de irrigação e drenagem de três localidades da Planície Costeira desse estado.

\section{Material e Métodos}

\section{1. Áreas de estudo}

O presente estudo foi realizado em três localidades da Planície Costeira do Rio Grande do Sul, Brasil, sendo uma na Planície Costeira externa à Lagoa dos Patos (Santo Antônio da Patrulha) e as outras duas, na Planície Costeira Interna (Cachoeirinha e Camaquã). Santo Antônio da Patrulha e Cachoeirinha pertencem à bacia hidrográfica do Guaíba (sub-bacias dos rios do Sinos e Gravataí, respectivamente) e Camaquã, à bacia hidrográfica do Litoral (sub-bacia do rio Camaquã) (Figura 1). A área de estudo em Cachoeirinha é composta por sete sítios, sendo três tipos de canais (canal de irrigação, canal de drenagem principal e canais de drenagem secundários em torno das lavouras) e quatro lavouras com diferentes técnicas (plantio direto, plantio convencional, plantio pré-germinado e cultivo mínimo). Para as lavouras e canais de drenagem foram delineadas três repetições, enquanto para os canais de irrigação e drenagem principal, os quais são únicos, foram feitas repetições em três pontos ao longo do canal (pseudo-repetições). Em Santo Antônio da Patrulha e Camaquã, foi possível selecionar três áreas em cada localidade, havendo, portanto, três repetições. Em cada área de amostragem nessas duas localidades, foram realizadas amostragens em um canal de irrigação, um canal de drenagem e uma lavoura com cultivo do tipo mínimo.

No Rio Grande do Sul, o ciclo anual de cultivo do arroz é iniciado usualmente em outubro, com a preparação dos campos de cultivo, e finalizado em março, quando ocorre a colheita. De novembro/ dezembro a fevereiro, as lavouras são mantidas irrigadas através do bombeamento de água para o interior do sistema. Em fevereiro, o fornecimento de água para as lavouras é interrompido, ocorrendo gradativa redução do nível de água nas lavouras e canais de drenagem secundários. O canal de irrigação e o canal de drenagem principal permanecem usualmente alagados. Entre os quatro principais tipos de cultivo utilizados no sul do Brasil (plantio direto, plantio convencional, plantio pré-germinado e cultivo mínimo), há variações quanto à época de plantio das sementes, duração da fase de inundação e manejo do solo antes e após a colheita, dentre outras (Embrapa 2005). 


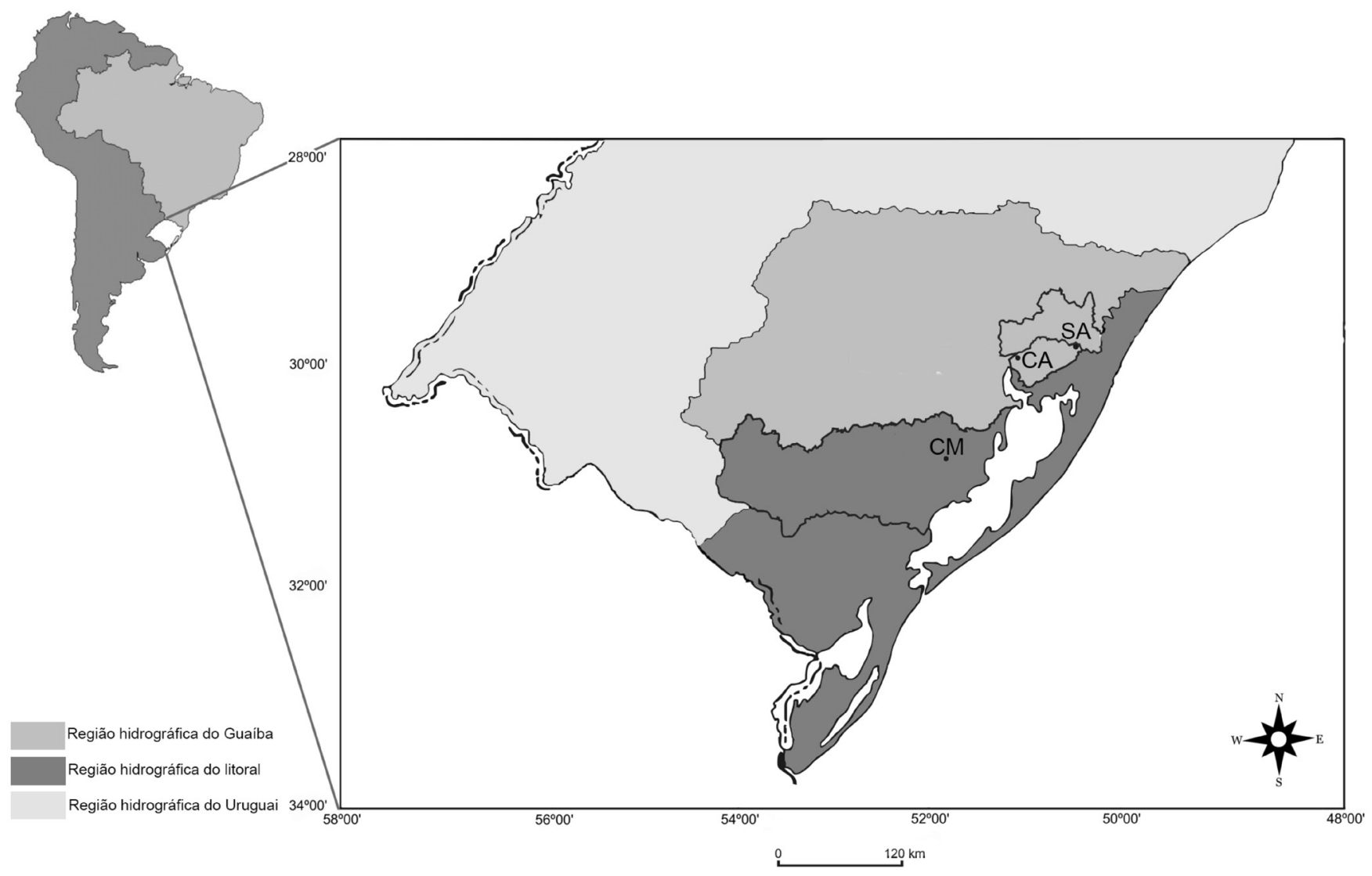

Figura 1. Áreas de estudo no Rio Grande do Sul, Brasil, e suas respectivas sub-bacias hidrográficas: Cachoeirinha (CA); Camaquã (CM); Santo Antônio da Patrulha (SA).

Figure 1. Study areas in southern Brazil and their respective river sub-basins: Cachoeirinha (CA); Camaquã (CM); Santo Antônio da Patrulha (SA).

A área localizada em Cachoeirinha corresponde à Estação Experimental do Arroz (EEA-IRGA) (29 $55^{\circ} 30^{\prime}$ 'S e $50^{\circ} 58^{\prime} 21^{\prime}$ ' O), a qual utiliza água oriunda do Rio Gravataí. No município de Santo Antônio da Patrulha, as três áreas investigadas situam-se entre as coordenadas $29^{\circ} 42^{\prime} 43^{\prime \prime} \mathrm{S}$; $50^{\circ} 38^{\prime} 18^{\prime \prime} \mathrm{O}(\mathrm{SA} 1), 29^{\circ} 55^{\prime} 07^{\prime \prime} \mathrm{S}$; $50^{\circ} 40^{\prime} 38^{\prime \prime} \mathrm{O}(\mathrm{SA} 2)$ e $29^{\circ} 52^{\prime} 41^{\prime \prime} \mathrm{S}$ e $58^{\circ} 37^{\prime} 27^{\prime \prime} \mathrm{O}$ (SA3). A área SA1 é irrigada com água do alto rio dos Sinos. Para irrigação da área SA2 a água é retirada do rio Gravataí. A área SA3 é irrigada com água proveniente de dois arroios, Pitangueira e Passo dos Ramos que são afluentes do rio Gravataí e do Açude dos Pereiras. As três áreas de estudo localizadas no município de Camaquã, situadas, respectivamente, entre as coordenadas $30^{\circ} 49^{\prime} 97^{\prime \prime} \mathrm{S} ; 51^{\circ} 50^{\prime} 47^{\prime \prime}$

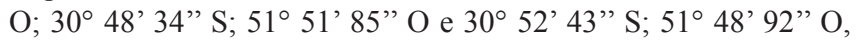
são irrigadas por meio de canais de irrigação com água oriunda da represa do arroio Duro.

\section{Amostragens}

As amostragens de turbelários foram realizadas mensalmente nos ambientes permanentemente alagados (canal de irrigação e canal de drenagem principal) das áreas de estudo. Nas lavouras e/ou canais de drenagem que circundam as parcelas (canais de drenagem secundários), as amostragens foram feitas no período de inundação desses ambientes, usualmente de dezembro a fevereiro. Em Cachoeirinha, as amostragens foram realizadas de dezembro de 2006 a junho de 2010, incluindo três ciclos de cultivo e entressafras, e, em Santo Antônio da Patrulha e Camaquã, de agosto de 2008 a agosto de 2009, correspondentes a um ciclo de cultivo e entressafras. Para as amostragens, utilizou-se puçá com malha de $335 \mu \mathrm{m}$, conforme metodologia empregada por Gamo \& Leal-Zanchet (2004), sendo que o esforço amostral foi padronizado em dez minutos para cada um dos pontos de coleta (sítios), com três repetições. Amostras de água, com o conteúdo do puçá, foram levadas ao laboratório para triagem.

\section{Triagem e determinação taxonômica}

As amostras foram inicialmente examinadas a olho nu, para localização dos espécimes, durante dois a três dias, sendo contados todos os espécimes visualizados (abundância de espécimes). Estes foram isolados com auxílio de pipeta e analisados, vivos, com auxílio de estereomicroscópio. A análise da morfologia externa foi complementada ao microscópio óptico (Young 2001). Posteriormente, cada espécime foi submetido à técnica de squash progressivo para análise da morfologia interna. Com fins de determinação taxonômica, os turbelários foram, adicionalmente, desenhados, fotografados, medidos com lâmina micrométrica e identificados com o auxílio de chaves dicotômicas. Os animais identificados apenas em nível de morfoespécie foram fixados em SUSA ou formalina neutra para processamento histológico. As preparações histológicas desses animais foram incluídas na Coleção Científica de Platyhelminthes do Museu de Zoologia da UNISINOS.

\section{Análise dos dados}

Para cada localidade, foi calculada a abundância total de espécimes nas amostragens. A abundância relativa foi obtida considerando a porcentagem de indivíduos de cada grupo taxonômico em relação ao total de turbelários amostrados. 


\section{Resultados}

Nas áreas de estudo, registrou-se um total de 144 espécies de turbelários, sendo 141 microturbelários e três macroturbelários (Tabelas 1,2). As espécies distribuem-se em seis grupos taxonômicos principais (Catenulida, Macrostomida, Lecithoepitheliata, Proseriata, Rhabdocoela e Tricladida) (Tabelas 2, 3). Vinte e três espécies são registradas pela primeira vez para o Rio Grande do Sul, seis delas representando também o primeiro registro para o país (Tabela 2).

$\mathrm{O}$ maior número de espécies de turbelários foi registrado em Cachoeirinha, sendo também a localidade que apresentou maior

Tabela 1. Total de registros de espécies de turbelários e total de espécimes amostrados por município em lavouras de arroz e canais de irrigação e drenagem em três localidades da Planície Costeira do Rio Grande do Sul, Brasil. *Espécies comuns entre as localidades foram contadas uma única vez para o total de espécies registradas.

Table 1. Total of recorded species of turbellarians and total of sampled specimens per locality in rice fields and their irrigation and drainage canals in three areas of the Coastal Plain, in Rio Grande do Sul, Brazil. *Species occurring in two or more localities were counted once to obtain the amount of recorded species.

\begin{tabular}{lccc}
\hline Localidades & $\begin{array}{c}\text { Número } \\
\text { de espécies } \\
\text { registradas }\end{array}$ & $\begin{array}{c}\text { Total de espécimes } \\
\text { amostrados por } \\
\text { localidade }\end{array}$ & $\begin{array}{c}\text { Espécies } \\
\text { únicas }\end{array}$ \\
\hline Cachoeirinha & 121 & 15379 & 35 \\
Santo Antônio & 88 & 13470 & 9 \\
Camaquã & 73 & 5454 & 11 \\
Total & $144^{*}$ & 34303 & 55 \\
\hline
\end{tabular}

abundância absoluta (Tabela 1). Em Cachoeirinha, Catenula lemnae Duges, 1832 foi o microturbelário com maior abundância relativa, seguido de Bothromesostoma personatum (Schmidt, 1848), Stenostomum tenuicauda Nuttycombe \& Waters, 1938 e Stenostomum bicaudatum Kennel, 1888. Adicionalmente, os macroturbelários Girardia sp. e Rhodax sp. estiveram representados dentre aqueles com abundância relativa alta em Cachoeirinha. Girardia tigrina (Girard, 1850) foi a espécie com maior abundância relativa em Santo Antônio da Patrulha e em Camaquã, representando também o turbelário com maior número de espécimes coletados no estudo (Tabela 2). Em Santo Antônio, outras espécies com abundância relativa alta foram Mesostoma platycephalum Braun, 1885, C. lemnae, Stenostomum sp.9 e Microstomum lineare (Müller, 1773), enquanto, em Camaquã, S. bicaudatum, Dasyhormus lithophorus Marcus, 1945, Anokkostenostomum pseudoacetabulum (Nuttycombe \& Waters, 1935) e S. tenuicauda (Tabela 2). Do total de espécies, cerca de $38 \%$ foram registradas exclusivamente numa das três localidades (Tabela 2). Aproximadamente $28 \%$ das espécies foram registradas nas três áreas de estudo. Algumas dessas são espécies cosmopolitas que já haviam sido registradas para a América do Sul, tais como $C$. lemnae, G. tigrina, P. stagnalis Schultze, 1851, B. semperi Braun, 1881 e Gyratrix hermaphroditus Ehrenberg, 1831, dentre outras.

Dos seis principais grupos taxonômicos registrados no presente estudo, Rhabdocoela, Catenulida e Macrostomida contribuíram com o maior número de espécies novas, a maioria das quais foi identificada como morfoespécie (Tabelas 2, 3). Catenulida representou o grupo com maior abundância relativa em Cachoeirinha, sendo seguido por Rhabdocoela. Nas outras duas localidades, a maior abundância relativa foi de Tricladida, seguido por Catenulida (Tabela 4). Não houve registros de indivíduos de Proseriata para Cachoeirinha (Tabelas 3,4).

Tabela 2. Espécies de turbelários registradas e abundância absoluta e relativa por espécie em cada localidade em ecossistemas de arroz irrigado da Planície Costeira do Rio Grande do Sul, Brasil. ${ }^{1}$ Novos registros para o estado do Rio Grande do Sul; ${ }^{2}$ Novos registros para o Brasil.

Table 2. Turbellarian species occurring in irrigated rice fields and absolute and relative abundance per species in each studied area in rice fields and their irrigation and drainage canals of the Coastal Plain, in Rio Grande do Sul, Brazil. ${ }^{1}$ New records for the state of Rio Grande do Sul; ${ }^{2}$ New records for Brasil.

\begin{tabular}{|c|c|c|c|c|c|c|}
\hline & \multicolumn{2}{|c|}{ Cachoeirinha } & \multicolumn{2}{|c|}{ Santo Antônio } & \multicolumn{2}{|c|}{ Camaquã } \\
\hline & $\begin{array}{c}\text { Abundância } \\
\text { Absoluta }\end{array}$ & $\begin{array}{c}\text { Abundância } \\
\text { Relativa }\end{array}$ & $\begin{array}{c}\text { Abundância } \\
\text { Absoluta }\end{array}$ & $\begin{array}{c}\text { Abundância } \\
\text { Relativa }\end{array}$ & $\begin{array}{c}\text { Abundância } \\
\text { Absoluta }\end{array}$ & $\begin{array}{c}\text { Abundância } \\
\text { Relativa }\end{array}$ \\
\hline \multicolumn{7}{|l|}{ CATENULIDA } \\
\hline $\begin{array}{l}\text { Anokkostenostomum anatirostrum } \\
\text { (Marcus, 1945) }\end{array}$ & - & - & 1 & 0,01 & 1 & 0,02 \\
\hline $\begin{array}{l}\text { Anokkostenostomum membranosum } \\
(\text { Kepner \& Carter, 1931) }\end{array}$ & 6 & 0,04 & - & - & - & - \\
\hline $\begin{array}{l}\text { Anokkostenostomum pseudoacetabulum } \\
\text { (Nuttycombe \& Waters, 1935) }\end{array}$ & 21 & 0,14 & 15 & 0,11 & 241 & 4,21 \\
\hline $\begin{array}{l}\text { Anokkostenostomum saliens } \\
\text { (Kepner \& Carter, 1931) }^{1}\end{array}$ & 3 & 0,02 & - & -- & 1 & 0,02 \\
\hline $\begin{array}{l}\text { Anokkostenostomum tuberculosum } \\
\text { (Nuttycombe \& Waters, 1938) }\end{array}$ & 79 & 0,51 & 57 & 0,42 & 6 & 0,10 \\
\hline Catenula lemnae Duges, 1832 & 7115 & 46,26 & 515 & 3,82 & 1 & 0,02 \\
\hline Chordarium leucanthum Marcus, $1945^{1}$ & - & - & 1 & 0,01 & - & - \\
\hline Dasyhormus lithophorus Marcus, $1945^{1}$ & - & - & 277 & 2,06 & 277 & 4,84 \\
\hline Stenostomum amphotum Marcus, 1945 & 246 & 1,60 & 62 & 0,46 & 33 & 0,58 \\
\hline Stenostomum arevaloi Gieysztor, $1931^{1}$ & 25 & 0,16 & 11 & 0,08 & 69 & 1,21 \\
\hline Stenostomum bicaudatum Kennel, 1888 & 550 & 3,58 & 311 & 2,31 & 407 & 7,11 \\
\hline Stenostomum ciliatum Kepner \& Carter, $1931^{1}$ & 3 & 0,02 & 1 & 0,01 & 1 & 0,02 \\
\hline
\end{tabular}


Tabela 2. Continuação...

Table 2. Continued...

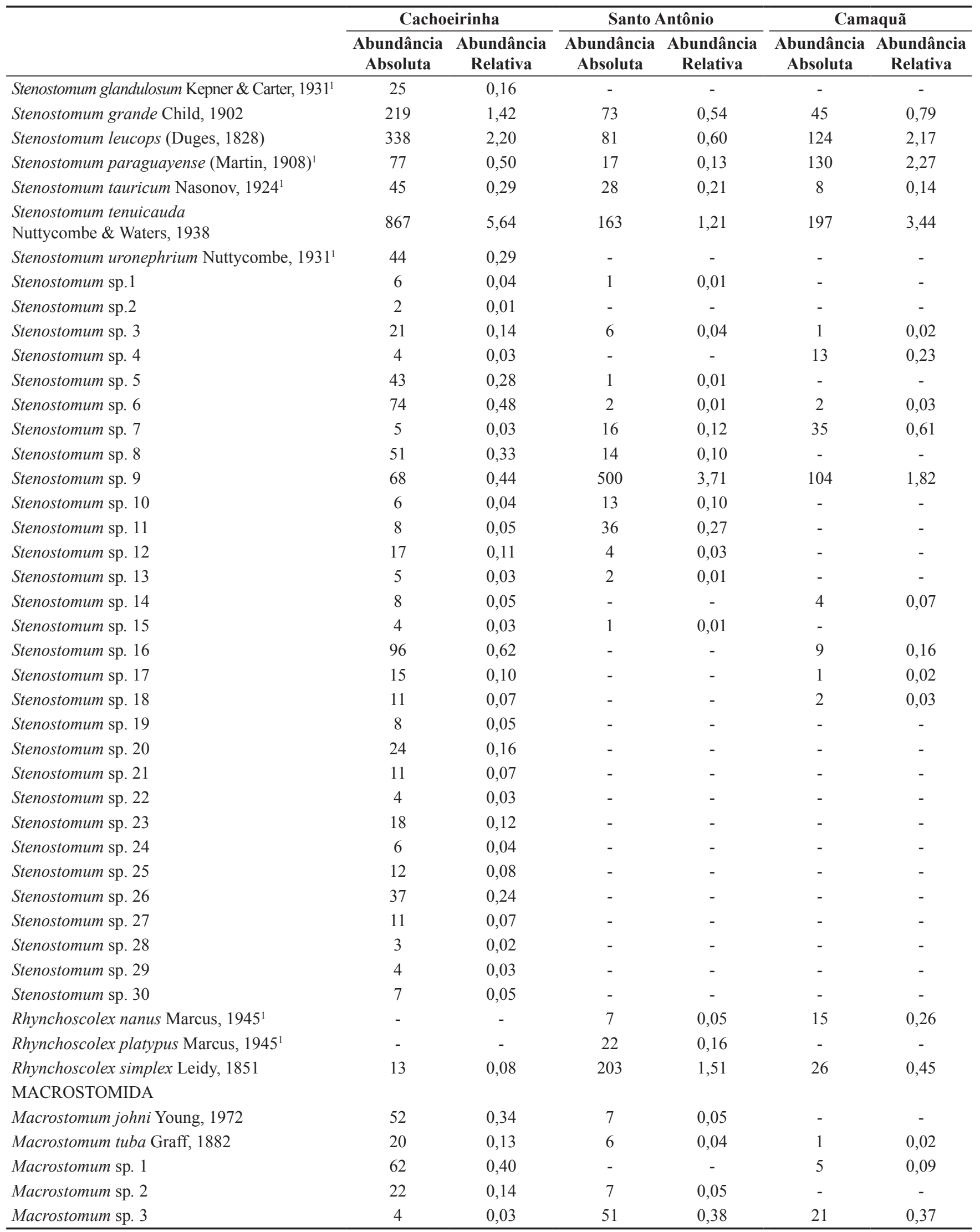


Tabela 2. Continuação...

Table 2. Continued...

\begin{tabular}{|c|c|c|c|c|c|c|}
\hline & \multicolumn{2}{|c|}{ Cachoeirinha } & \multicolumn{2}{|c|}{ Santo Antônio } & \multicolumn{2}{|c|}{ Camaquã } \\
\hline & $\begin{array}{c}\text { Abundância } \\
\text { Absoluta }\end{array}$ & $\begin{array}{c}\text { Abundância } \\
\text { Relativa }\end{array}$ & $\begin{array}{c}\text { Abundância } \\
\text { Absoluta }\end{array}$ & $\begin{array}{c}\text { Abundância } \\
\text { Relativa }\end{array}$ & $\begin{array}{c}\text { Abundância } \\
\text { Absoluta }\end{array}$ & $\begin{array}{c}\text { Abundância } \\
\text { Relativa }\end{array}$ \\
\hline Macrostomum sp. 4 & 6 & 0,04 & 5 & 0,04 & - & - \\
\hline Macrostomum sp. 5 & 6 & 0,04 & 7 & 0,05 & - & - \\
\hline Macrostomum sp. 6 & 14 & 0,09 & 52 & 0,39 & 28 & 0,49 \\
\hline Macrostomum sp. 7 & 28 & 0,18 & 88 & 0,65 & 2 & 0,03 \\
\hline Macrostomum sp. 8 & 5 & 0,03 & - & - & 1 & 0,02 \\
\hline Macrostomum sp. 9 & 16 & 0,10 & 11 & 0,08 & - & - \\
\hline Macrostomum sp. 10 & 52 & 0,34 & 56 & 0,42 & 146 & 2,55 \\
\hline Macrostomum sp. 11 & 27 & 0,18 & 1 & 0,01 & - & - \\
\hline Macrostomum sp. 12 & - & - & 14 & 0,10 & 1 & 0,02 \\
\hline Macrostomum sp. 13 & - & - & 10 & 0,07 & 5 & 0,09 \\
\hline Macrostomum sp. 14 & - & - & 37 & 0,27 & - & - \\
\hline Macrostomum sp. 15 & - & - & 1 & 0,01 & - & - \\
\hline Macrostomum sp. 16 & 12 & 0,08 & - & - & - & - \\
\hline Macrostomum sp. 17 & 11 & 0,07 & - & - & - & - \\
\hline Macrostomum sp. 18 & 19 & 0,12 & - & - & - & - \\
\hline Microstomum lineare (Müller, 1773) & 415 & 2,70 & 342 & 2,54 & 134 & 2,34 \\
\hline \multicolumn{7}{|l|}{ LECITHOEPITHELIATA } \\
\hline Geocentrophora sp.1 & 15 & 0,10 & 14 & 0,10 & - & - \\
\hline Geocentrophora sp.2 & - & - & 1 & 0,01 & - & - \\
\hline Prorhynchus stagnalis Schultze, 1851 & 45 & 0,29 & 108 & 0,80 & 29 & 0,51 \\
\hline Lecithoepitheliata 1 & - & - & 5 & 0,04 & - & - \\
\hline \multicolumn{7}{|l|}{ PROSERIATA } \\
\hline Bothrioplana semperi Braun, $1881^{1}$ & - & - & 1 & 0,01 & 2 & 0,03 \\
\hline \multicolumn{7}{|l|}{ RHABDOCOELA: Dalyellioida } \\
\hline Dalyellioida 1 & 23 & 0,15 & - & - & 3 & 0,05 \\
\hline Dalyellioida 2 & 32 & 0,21 & 4 & 0,03 & - & - \\
\hline Dalyellioida 3 & 15 & 0,10 & 5 & 0,04 & 6 & 0,10 \\
\hline Dalyellioida 4 & 184 & 1,20 & 19 & 0,14 & 7 & 0,12 \\
\hline Dalyellioida 5 & 358 & 2,33 & - & - & 2 & 0,03 \\
\hline Dalyellioida 6 & 3 & 0,02 & - & - & 28 & 0,49 \\
\hline Dalyellioida 7 & 13 & 0,08 & 1 & 0,01 & 9 & 0,16 \\
\hline Dalyellioida 8 & 7 & 0,05 & 1 & 0,01 & - & - \\
\hline Dalyellioida 9 & 4 & 0,03 & - & - & 3 & 0,05 \\
\hline Dalyellioida 10 & 6 & 0,04 & - & - & 1 & 0,02 \\
\hline Dalyellioida 11 & 11 & 0,07 & - & - & 1 & 0,02 \\
\hline Dalyellioida 12 & 17 & 0,11 & 7 & 0,05 & - & - \\
\hline Dalyellioida 13 & 58 & 0,38 & 22 & 0,16 & - & - \\
\hline Dalyellioida 14 & 21 & 0,14 & 19 & 0,14 & 4 & 0,07 \\
\hline Dalyellioida 15 & 38 & 0,25 & 4 & 0,03 & 5 & 0,09 \\
\hline Dalyellioida 16 & 9 & 0,06 & 37 & 0,27 & 66 & 1,15 \\
\hline Dalyellioida 17 & 23 & 0,15 & 1 & 0,01 & - & - \\
\hline Dalyellioida 18 & - & - & 124 & 0,92 & 1 & 0,02 \\
\hline Dalyellioida 19 & - & - & - & - & 1 & 0,02 \\
\hline Dalyellioida 20 & - & - & - & - & 1 & 0,02 \\
\hline Dalyellioida 21 & 18 & 0,12 & - & - & - & - \\
\hline Gieysztoria trisolena (Marcus, 1946) & 8 & 0,05 & - & - & - & - \\
\hline Gieysztoria rubra (Fuhrmann, 1894) ${ }^{2}$ & 8 & 0,05 & - & - & - & - \\
\hline
\end{tabular}


Tabela 2. Continuação...

Table 2. Continued..

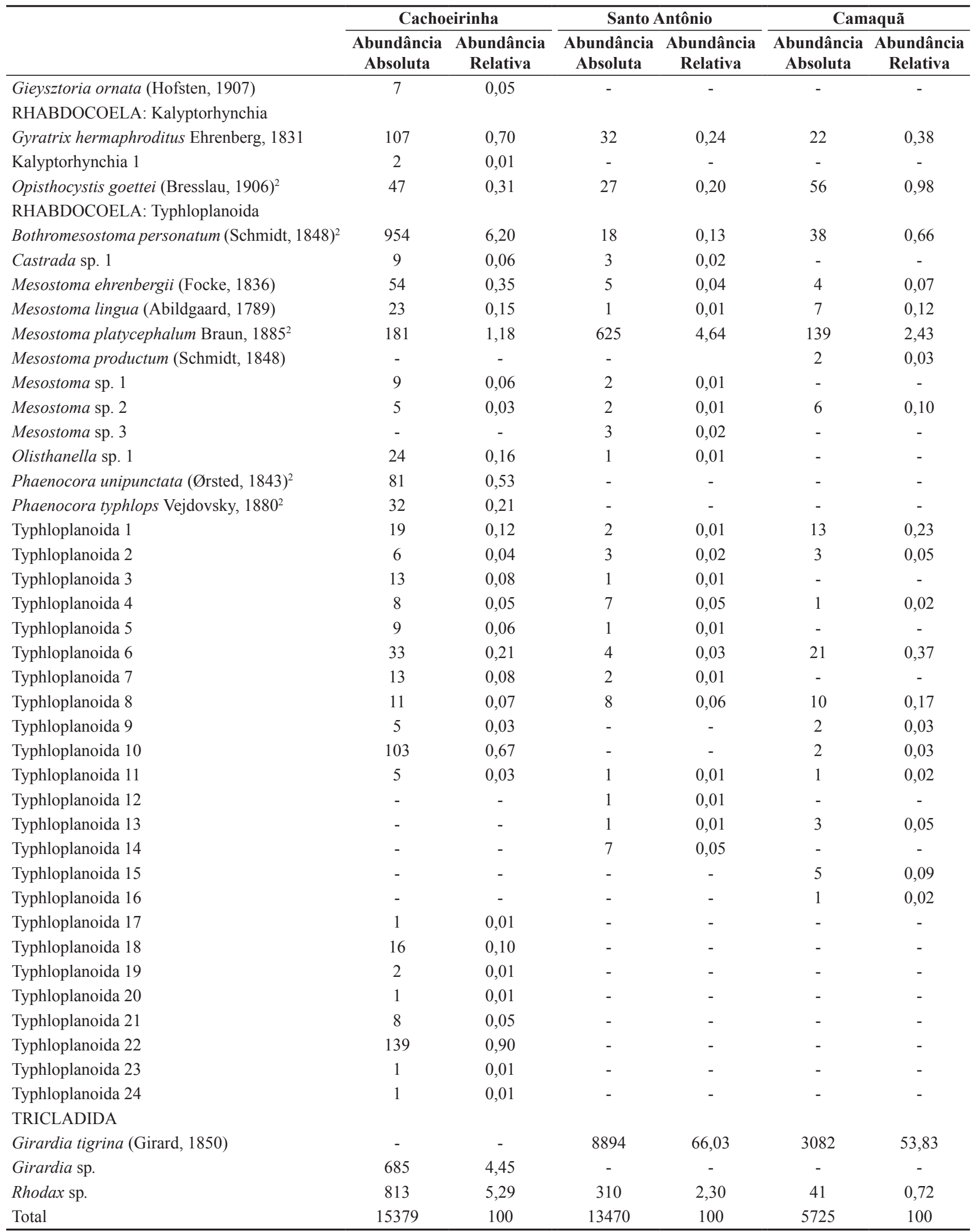




\section{Discussão}

Neste estudo foram registradas 144 espécies de turbelários para três localidades do sul do Brasil (Cachoeirinha, Santo Antônio e Camaquã), o que pode ser considerado um número elevado, tendo em vista o total de 98 espécies registradas em ambientes dulcícolas no Brasil (Carbayo et al. 2009). Grande parte dos espécimes foi identificado como morfoespécie, a maioria delas representando espécies novas para a ciência. Além disso, algumas morfoespécies estiveram representadas por poucos indivíduos ou espécimes sem aparelho copulador, dificultando a análise de características necessárias para identificação ou, ainda, devido à necessidade de realizar análise comparativa detalhada com todas as espécies descritas para cada gênero ou família. Dificuldades similares de identificação em nível de espécie têm ocorrido com os tricladidos terrestres, quando da realização de inventários ou de estudos de estrutura de comunidades (Leal-Zanchet \& Carbayo 2000, Carbayo et al. 2002, Castro \& Leal-Zanchet 2005, Baptista et al. 2006, 2010, Fick et al. 2006, Antunes et al. 2008, Leal-Zanchet et al. 2011). Ao contrário dos macroturbelários, investigados mais amplamente no estado, os microturbelários foram estudados apenas recentemente, tendo sido registrada inicialmente a ocorrência de dez espécies dulciaquícolas para o estado, pertencentes a três grupos taxonômicos, Catenulida, Macrostomida e Rhabdocoela (Gamo \& Leal-Zanchet 2004). Posteriormente, Braccini \& Leal-Zanchet (2013) registraram 40 espécies de microturbelários, diversas identificadas apenas como morfoespécie, pertencentes aos grupos taxonômicos Catenulida,

Tabela 3. Total de espécies de turbelários registradas por grupo taxonômico em ecossistemas de arroz irrigado da Planície Costeira do Rio Grande do Sul, Brasil. *Espécies comuns entre as localidades foram contadas uma única vez para o total de espécies registradas.

Table 3. Total of recorded species of turbellarians per taxonomic group in irrigated rice fields of the Coastal Plain, in Rio Grande do Sul, Brazil. * Species occurring in two or more localities were counted once to obtain the amount of recorded species.

\begin{tabular}{lcccc}
\hline & Cachoeirinha & $\begin{array}{c}\text { Santo } \\
\text { Antônio }\end{array}$ & Camaquã & Total* \\
\hline Catenulida & 47 & 30 & 26 & 52 \\
Macrostomida & 17 & 16 & 10 & 21 \\
Rhabdocoela & 53 & 35 & 34 & 63 \\
Lecithoepitheliata & 2 & 4 & 1 & 4 \\
Proseriata & - & 1 & 1 & 1 \\
Tricladida & 2 & 2 & 2 & 3 \\
Total & 121 & 88 & 74 & 144 \\
\hline
\end{tabular}

Macrostomida, Lecithoepitheliata e Rhabdocoela, e duas espécies de macroturbelários (Tricladida) em lagoas costeiras do Rio Grande do Sul. O presente trabalho amplia o número de espécies de turbelários registradas para o estado do RS, de 29 para 52. Os espécimes identificados como morfoespécie, num total de 101 correspondem, na sua maioria, a espécies ainda não descritas, indicando a ocorrência de um alto número de espécies novas nas áreas de estudo.

A ocorrência de determinadas espécies em apenas uma das áreas de estudo indica o grau de especificidade de hábitat desses organismos. Resultados similares têm sido verificados para os tricladidos terrestres (Baptista et al. 2006, 2010, Fick et al. 2006, Antunes et al. 2008, Leal-Zanchet et al. 2011). No entanto, há um elevado número de espécies com baixa abundância, em cada localidade, de forma que o fato de não terem sido amostradas em um determinado local pode ser devido à ocorrência de poucos indivíduos. Como os arrozais são ambientes heterogêneos, tanto em escala espacial como temporal, os turbelários provavelmente selecionam microhábitats que favoreçam a sua sobrevivência e minimizem o estresse decorrente dessas oscilações. No caso dos microturbelários, muitas espécies apresentam rápido desenvolvimento e reprodução assexuada, tais como Catenula lemnae, Stenostomum tenuicauda, Rhodax sp. e Stenostomum bicaudatum, enquanto os rabdocelos produzem ovos resistentes que persistem no ambiente, em condições adversas, tais como períodos de seca (Young 2001), de forma que espécies com tais características têm maior probabilidade de ocorrerem nesse tipo de ambiente.

No presente estudo, obteve-se um grande número de registros de turbelários em lavouras de arroz irrigado e seus canais de irrigação e drenagem. No entanto, não há, no Brasil, estudos extensivos da diversidade de turbelários em áreas úmidas naturais que possam ser utilizados para comparação com os resultados aqui obtidos, uma vez que as amostragens realizadas para estudos taxonômicos desenvolvidos especialmente em São Paulo e no Rio Grande do Sul foram pontuais. A análise da estrutura de comunidades de turbelários em lagoas costeiras, realizada por Braccini \& Leal-Zanchet (2013), ao longo de um ciclo anual, baseou-se em um número menor de amostragens do que o presente estudo. $\mathrm{O}$ elevado número de espécies registradas e de registros de determinadas espécies em arrozais irrigados reflete a habilidade dos turbelários colonizarem áreas úmidas temporárias. Contudo, os agroecossistemas orizícolas apresentam condições adversas que podem limitar a sobrevivência de determinadas espécies de turbelários. Características como hidroperíodo, permanência de baixa lâmina d'água, manejo mecânico e adição de biocidas e fertilizantes podem atuar como determinantes da sobrevivência de poucos espécimes de turbelários, ou seja, de espécies que apresentam maior tolerância a essas alterações típicas dos agrossistemas de cultivo de arroz irrigado. A permanência de baixa

Tabela 4. Abundância absoluta e relativa de turbelários por grupo taxonômico em cada localidade em ecossistemas de arroz irrigado da Planície Costeira do Rio Grande do Sul, Brasil.

Table 4. Absolute and relative abundance of turbellarians per taxonomic group and per municipality in irrigated rice fields of the Coastal Plain, in Rio Grande do Sul, Brazil.

\begin{tabular}{|c|c|c|c|c|c|c|}
\hline & \multicolumn{2}{|c|}{ Cachoeirinha } & \multicolumn{2}{|c|}{ Santo Antônio } & \multicolumn{2}{|c|}{ Camaquã } \\
\hline & $\begin{array}{c}\text { Abundância } \\
\text { Absoluta }\end{array}$ & $\begin{array}{c}\text { Abundância } \\
\text { Relativa }\end{array}$ & $\begin{array}{c}\text { Abundância } \\
\text { Absoluta }\end{array}$ & $\begin{array}{c}\text { Abundância } \\
\text { Relativa }\end{array}$ & $\begin{array}{c}\text { Abundância } \\
\text { Absoluta }\end{array}$ & $\begin{array}{c}\text { Abundância } \\
\text { Relativa }\end{array}$ \\
\hline CATENULIDA & 10265 & 66,75 & 2441 & 18,12 & 1753 & 30,62 \\
\hline MACROSTOMIDA & 771 & 5,01 & 695 & 5,16 & 344 & 6,01 \\
\hline LECITHOEPITHELIATA & 60 & 0,39 & 128 & 0,95 & 29 & 0,51 \\
\hline PROSERIATA & 0 & 0 & 1 & 0,01 & 2 & 0,03 \\
\hline RHABDOCOELA & 2785 & 18,11 & 1001 & 7,43 & 474 & 8,28 \\
\hline TRICLADIDA & 1498 & 9,74 & 9204 & 68,33 & 3123 & 54,55 \\
\hline Total & 15379 & 100 & 13470 & 100 & 5725 & 100 \\
\hline
\end{tabular}


lâmina d'água, por si só, provavelmente não afeta a sobrevivência da maioria das espécies, mas o hidroperíodo nas lavouras representa um fator limitante para muitas espécies de turbelários que não apresentam capacidade de produção de ovos resistentes à dessecação, o que ocorre apenas nos rabdocelos. No entanto, a colonização desses ambientes pode ocorrer rapidamente, como em outros ambientes temporários, devido ao ciclo de vida versátil dos turbelários, incluindo a capacidade de reprodução assexuada.

\section{Agradecimentos}

Ao $\mathrm{CNPq}$, a bolsa de produtividade em pesquisa e o apoio financeiro concedido ao projeto de pesquisa $\mathrm{n}^{\circ}$. 478903/2010-3. Ao Instituto Rio Grandense do Arroz, a bolsa concedida a DCV e o apoio logístico para as amostragens em Camaquã e Santo Antônio da Patrulha. Aos pesquisadores Vera Mussoi Macedo e Élio Marcolin, do Instituto Rio Grandense do Arroz, o apoio para desenvolvimento desse estudo. Aos senhores Luiz Carlos Machado, Jorge Luiz dos Santos Dutra e Cícero Borges da Cunha, proprietários das lavouras, por possibilitarem o acesso às áreas de estudo de Santo Antônio da Patrulha. À Associação dos Usuários do Perímetro de Irrigação do Arroio Duro (AUD), de Camaquã, pela disponibilização de áreas amostrais para realização de parte desse estudo. A Daniela Rocha, Denise Cristina Borges, Julia Klee Noll, Juliana Alves de Espíndola e Silvana Vargas do Amaral, o auxílio nas coletas. Às laboratoristas Letícia Ayres Guterrez, Aline Felten Centa e Vanessa dos Anjos Batista, o processamento histológico e a manutenção dos animais. A Silvana Vargas do Amaral, a elaboração da versão final da Figura 1. A dois revisores anônimos, as sugestões ao manuscrito.

\section{Referências Bibliográficas}

AMATO, J.F.R. \& AMATO, S.B. 2005. New species of Temnocephala Blanchard (Platyhelminthes, Temnocephalida) ectosymbiont on giant water bugs, Belostoma spp. (Hemiptera, Belostomatidae) from southern Brazil. Rev. Bras. Zool. 22(1):107-118. http://dx.doi.org/10.1590/S010181752005000100014

AMATO, J.F.R., AMATO, S.B. \& DAUDT, L.C.C. 2003. New species of Temnocephala Blanchard (Platyhelminthes, Temnocephalida) ectosymbiont on Aegla serrana Buckup \& Rossi (Crustacea, Anomura) from southern Brazil. Rev. Bras. Zool. 20(3):493-500. http://dx.doi. org/10.1590/S0101-81752003000300021

AMATO, J.F.R., AMATO, S.B. \& SEIXAS, S.A. 2005. Temnocephala lutzi Monticelli (Platyhelminthes, Temnocephalida) ectosymbiont on two species of Trichodactylus Latreille (Crustacea, Decapoda, Trichodactylidae) from southern Brazil. Rev. Bras. Zool. 22(4):10851094. http://dx.doi.org/10.1590/S0101-81752005000400038

AMATO, J.F.R., AMATO, S.B. \& SEIXAS, S.A. 2006. New species of Temnocephala Blanchard (Platyhelminthes, Temnocephalida) ectosymbiont on Trichodactylus fluviatilis Latreille (Crustacea, Decapoda, Trichodactylidae) from southern Brazil. Rev. Bras. Zool. 23(3):796-806. http://dx.doi.org/10.1590/S0101-81752006000300026

AMATO, J.F.R., SEIXAS, S.A. \& AMATO, S.B. 2007. New species of Temnocephala Blanchard (Platyhelminthes, Temnocephalida) ectosymbiont on creeping water bugs, Cryphocrios granulosus De Carlo (Hemiptera, Naucoridae) from southern Brazil. Rev. Bras. Zool. 24(4):1043-1051. http://dx.doi.org/10.1590/S0101-81752007000400022

AMATO, J.F.R., AMATO, S.B. SEIXAS, S.A., FONSECA, M. \& ILÁRIO, R.J. 2010. Temnocephala pignalberiae Dioni, 1967 (Platyhelminthes, Temnocephalida) from two allopatric populations of Dilocarcinus pagei Stimpson, 1861 (Crustacea, Decapoda) first record for Brazil. Zootaxa 2613:15-28.

AMATO, J.F.R., AMATO, S.B., SEIXAS, S.A., VIDIGAL, T. \& ANDRADE, C.P. 2011. Trichoptera the newest insect order host of temnocephalans (Platyhelminthes, Temnocephalida) and the description of a new species of Temnocephala from Brazil. Zootaxa 2975:47-58.
ANTUNES, M.B., MARQUES, D.I.L. \& LEAL-ZANCHET, A.M. 2008. Composição das comunidades de planárias terrestres (Platyhelminthes, Tricladida, Terricola) em duas áreas de floresta estacional decidual do sul do Brasil. Neotrop. Biol. Conserv. 31:34-38.

BALL, I.R. \& REYNOLDSON, T.B. 1981. British planarians. Cambridge University Press, Cambridge.

BAMBARADENIYA, C.N.B., EDIRISINGHE, J.P., DE SILVA, D.N., GUNATILLEKE, C.V.S., RANAWANA, K.B. \& WIJEKOON, S. 2004. Biodiversity associated with an irrigated rice agro-ecosystem in Sri Lanka. Biodivers. Conserv. 13:1715-53. http://dx.doi.org/10.1023/ B:BIOC.0000029331.92656.de

BAPTISTA, V.A., MATOS, L.B., FICK, I.A. \& LEAL-ZANCHET, A.M. 2006. Composição das comunidades de planárias terrestres (Platyhelminthes, Tricladida, Terricola) do Parque Nacional dos Aparados da Serra. Iheringia, série Zoologia, 96(3):293-297. http://dx.doi.org/10.1590/ S0073-47212006000300004

BAPTISTA, V.A., OLIVEIRA, S.M. \& LEAL-ZANCHET, A.M. 2010 Inventário de planárias terrestres (Platyhelminthes, Tricladida) em remanescente de Floresta Estacional Decidual do sul do Brasil. Biota Neotrop. 10(2): http://www.biotaneotropica.org.br/ v10n2/en/abstract?i nventory+bn02110022010 (último acesso em 06/08/2012).

BLANCO, D.E., LÓPEZ-LANÚS, B., DIAS, R.A., AZPIROZ, A. \& RILLA, F. 2006. Uso de arroceras por chorlos y playeros migratorios en el sur de América del Sur. Implicancias de conservación y manejo. Wetlands International, Buenos Aires.

BRACCINI, J.A.L. \& LEAL-ZANCHET, A.M. 2013. Turbellarian assemblages in coastal lagoons in southern Brazil. Inv. Biol. 132(4): 305-314. http://dx.doi.org/10.1111/ivb.12032

CARBAYO, F., LEAL-ZANCHET, A.M. \& VIEIRA, E.M. 2002. Flatworms (Platyhelminthes: Tricladida: Terricola) diversity vs. man-induced disturbance in a subtropical rainforest from Southern Brazil. Biodivers. Conserv. 11:1091-1104. http://dx.doi.org/10.1023/A:1015865005604

CARBAYO, F., FROEHLICH, E.M., LEAL-ZANCHET, A.M. \& AMATO, S.B. 2009. Turbelários (Platyhelminthes). Estado da Arte e Perspectivas para a Zoologia no Brasil. UFPR, Curitiba.

CASTRO, R.A. \& LEAL-ZANCHET, A.M. 2005. Composição de comunidades de planárias terrestres (Platyhelminthes) em áreas de floresta estacional decidual e de campo na região central do Rio Grande do Sul, Brasil. Acta Biol. Leop. 27(3):147-150.

CZECH, H.A. \& PARSONS, K.C. 2002. Agricultural wetlands and waterbirds: A review. Waterbirds 25:56-65.

DIEGUES, A.C., coord. 1990. Inventário de áreas Úmidas do Brasil: versão preliminar. Progr. Pesq. e Cons. de áreas Úmidas no Brasil. Pró-Reitoria de Pesquisa USP, IUCN, Fundação Ford, São Paulo.

DU-BOIS REYMOND MARCUS, E. \& MARCUS, E. 1951. Contributions to the natural history of Brazilian Turbellaria. Comm. Zool. Mus. Hist. Nat. Montevideo 3(63):1-25.

ELPHICK, C.S. 2000. Functional equivalency between rice fields and seminatural wetland habitats. Conserv. Biol. 14(1):181-191. http://dx.doi. org/10.1046/j.1523-1739.2000.98314.x

ELPHICK, C.S. \& ORING, L.W. 2003. Conservation implications of flooding rice fields on winter waterbird communities. Agric. Ecos. Environ. 94(1):17-29. http://dx.doi.org/10.1016/S0167-8809(02)00022-1

EMBRAPA. 2005. Cultivo do Arroz Irrigado no Brasil. http:// sistemasdeproducao.cnptia.embrapa.br/FontesHTML/Arroz/ ArrozIrrigadoBrasil (último acesso em 19/09/2013).

FICK, I.A., LEAL-ZANCHET, A.M. \& VIEIRA, E.M. 2006. Community structure of land flatworms (PLATYHELMINTHES: TERRICOLA):comparisons between Araucaria and Atlantic forest in Southern Brazil. Inv. Biol. 125(4):306-313. http://dx.doi.org/10.1111/ j.1744-7410.2006.00062.x

FORATTINI, O.P., GOMES, A.C. \& KAKITANI, I. 1989. Observações sobre mosquitos Culicidae adultos em cultivo irrigado de arroz no Vale do Ribeira, Estado de São Paulo, Brasil. Rev. Saúde Pública. 23(4):307-312. http://dx.doi.org/10.1590/S0034-89101989000400006

GAMO, J. \& LEAL-ZANCHET, A.M. 2004. Freshwater microturbellarians (Platyhelminthes) from Rio Grande do Sul, Brazil. Rev. Bras. Zool. 21(4):897-903. http://dx.doi.org/10.1590/S0101-81752004000400026

GUADAGNIN, D.L. \& MALTCHIK, L. 2007. Habitat and Landscape Factors Associated with Neotropical Waterbird Occurrence and Richness in Wetland Fragments. Biodivers. Conserv. 16:1231-1244. http://dx.doi. org/10.1007/s10531-006-9127-5 
KAWAKATSU, M. 1989. Morphological, karyological and taxonomic studies of freshwater planarians from south Brazil. IX. The final report. Occ. Pub. Biol. Lab. Fuji Women's Coll. 21:1-4.

KAWAKATSU, M., HAUSER, J. \& FRIEDRICH, S.M.G. 1976. The freshwater planarians from South Brazil. Bull. Nat. Sci. Mus., Ser. A, Zool. 2:205-223.

KAWAKATSU, M., HAUSER, J. \& FRIEDRICH, S.M.G. 1980. Morphological, karyological and taxonomic studies of freshwater planarians from South Brazil. I. A history of those studies and a list of localities in the vicinities of São Leopoldo. Bull. Fuji Women's Coll. 18:129-151.

KAWAKATSU, M., HAUSER, J., FRIEDRICH, S.M.G. \& SOUZA LIMA, O.D. 1982. Morphological, karyological and taxonomic studies of freshwater planarians from South Brazil. III. Dugesia tigrina (Girard, 1850) and Dugesia schubarti (Marcus, 1946) from the vicinities of São Carlos, Estado de São Paulo (Turbellaria, Tricladida, Paludicola). Bull. Fuji Women's College 20:73-90.

KAWAKATSU, M., HAUSER, J. \& FRIEDRICH, S.M.G. $1983 \mathrm{a}$. Morphological, karyological and taxonomic studies of freshwater planarians from South Brazil. V. Dugesia tigrina (Girard, 1850) from município Botucatu, Estado de São Paulo, and Dugesia schubarti (Marcus, 1946) from the vicinity of São Paulo. Bull. Fuji Women's Coll. 21:147-163.

KAWAKATSU, M., HAUSER, J., FRIEDRICH, S.M.G. \& YAMAYOSHI, T. 1983b. Morphological, karyological and taxonomic studies of freshwater planarians from South Brazil. IV. Dugesia anderlani sp. nov. (Turbellaria, Tricladida, Paludicola), a new species from São Leopoldo in Estado de Rio Grande do Sul. Annot. Zool. Japoneses 56(3):96-208.

KAWAKATSU, M., OKI, I., TAMURA, S., YAMAYOSHI, T., HAUSER, J. \& FRIEDRICH, S.M.G. 1984. Morphological, karyological and taxonomic studies of freshwater planarians from south Brazil. VI. Dugesia schubarti (Marcus, 1946) from the vicinity of São Leopoldo, Estado de Rio Grande do Sul. (Turbellaria, Tricladida, Paludicola). Bull. Fuji Women's Coll. 22:45-62.

KAWAKATSU, M., HAUSER, J. \& FRIEDRICH, S.M.G. 1985. Morphological, karyological and taxonomic studies of freshwater planarians from south Brazil. VII. Supplementary notes on Dugesia schubarti (Marcus, 1946) from the vicinity of São Leopoldo, Estado de Rio Grande do Sul (Turbellaria, Tricladida, Paludicola). Bull. Fuji Women's Coll., 23:101-109.

KAWAKATSU, M., HAUSER, J. \& FRIEDRICH, S.M.G. 1986. Morphological, karyological and taxonomic studies of freshwater planarians from South Brazil. VIII. Four Dugesia species (D. tigrina, D. schubarti, D. anderlani, and D. ardnti) collected from several localities in Estado do Rio Grande do Sul (Turbellaria, Tricladida, Paludicola). Bull. Fuji Women's Coll. 24:41-62.

KAWAKATSU, M., HAUSER, J. \& PONCE DE LEÓN, R. 1992. Freshwater planarians from Uruguay and Rio Grande do Sul, Brazil: Dugesia ururiograndeana sp. nov. and Dugesia tigrina (Girard, 1850) (Turbellaria, Tricladida, Paludicola). Bull. Biogeogr. Soc. Japan 47:33-50.

KOLASA, J. 1991. Flatworms: Turbellaria and Nemertea. In Ecology and classification of North American freshwater invertebrates. (J.H. Thorpe \& A.P. Covich, ed.). Academic Press, San Diego, p.145-171.

LEAL-ZANCHET, A.M. \& CARBAYO, F. 2000. Fauna de planárias terrestres (Platyhelminthes, Tricladida, Terricola) da Floresta Nacional de São Francisco de Paula, RS, Brasil: Uma análise preliminar. Acta Biol. Leopold. 22(1):19-25.

LEAL-ZANCHET, A.M., BAPTISTA, V.A., CAMPOS, L.M. \& RAFFO, J.F. 2011. Spatial and temporal patterns of land flatworms assemblages in Brazilian Araucária forests. Invertebr. Biol. 130(1):25-33. http://dx.doi. org/10.1111/j.1744-7410.2010.00215.x

MAEDA, T. 2001. Patterns of bird abundance and habitat use in rice fields of the Kanto Plain, central Japan. Ecol. Res. 16(3):569-585. http://dx.doi. org/10.1046/j.1440-1703.2001.00418.x

MALTCHIK, L., COSTA, E.S., BECKER, C.G. \& OLIVEIRA, A.E. 2003. Inventory of wetlands of Rio Grande do Sul (Brazil). Pesqui., Bot. 53:89-100.
MARCUS, E. 1943. O turbelário Mesostoma ehrenbergii Focke 1836 no Brasil. Bol. Indust. Animal 6:12-15.

MARCUS, E. 1944. Sobre duas Prorhynchidae (Turbellaria), novas para o Brasil. Arq. Mus. Paran. Curitiba 4:3-46.

MARCUS, E. 1945a. Sobre Catenulida Brasileiros. Bol. Fac. Filos. Cienc. Let. Univ. São Paulo 10:3-133.

MARCUS, E. 1945b. Sobre microturbellarios do brasil. Comun. Zool. Mus. Hist. Nat. Montevideo 1(25):1-74+4pl.

MARCUS, E. 1946. Sobre Turbellaria brasileiros. Bol. Fac. Filos. Cienc. Let. Univ. São Paulo 11:5-187+31pl.

MARCUS, E. 1948. Turbellaria do Brasil. Bol. Fac. Filos. Cienc. Let. Univ. São Paulo 13:111-243.

MARCUS, E. 1949. Turbellaria Brasileiros (7). Bol. Fac. Filos. Cienc. Let. Univ. São Paulo 14:7-156.

MARCUS, E. 1950. Turbellaria Brasileiros. Bol. Fac. Fil. Ciênc. Letr. Univ. São Paulo, Série Zoologia, 15: 5-192.

MARCUS, E. 1952. Turbellaria Brasileiros. Bol. Fac. Fil. Ciênc. Letr. Univ. São Paulo, Série Zoologia, 17: 5-187.

MARCUS, E. 1954. Turbellaria Brasileiros (11). Pap. Av. Dep. Zool. Secr. Agric. São Paulo 11(24):419-489.

NOREÑA-JANSSEN, C. 1995. Studies on the taxonomy and ecology of the turbellarian (Plathelminthes) in the floodplain of the Paraná river (Argentina). II. Taxonomy and ecology of the Turbellaria. Arch. Hydrobiol. Supp. 107:11-262.

NOREÑA, C., BRUSAF. \& FAUBEL, A. 2003. Census of "Microturbellarians" (free-living Platyhelminthes) of the zoogeographical regions originating from Gondwana. Zootaxa 146:1-34.

SCHOCKAERT, E.R., HOOGE, M., SLUYS, R., SCHILLING, S., TYLER, S. \& ARTOIS, T. 2008. Global diversity of free living flatworms (Platyhelminthes, "Turbellaria") in freshwater. Hydrobiologia 595:41-48. http://dx.doi.org/10.1007/s10750-007-9002-8

SEIXAS, S.A., AMATO, J.F.R. \& AMATO, S.B. 2011. A new species of Temnocephala Blanchard (Platyhelminthes, Temnocephalida) ectosymbiont on Dilocarcinus septemdentatus (Decapoda, Trichodactylidae) from the Brazilian Amazonia. Neotrop. Helminth. 5:200-211.

SEIXAS, S.A., AMATO, J.F.R. \& AMATO, S.B. 2010a. Redescription of Temnocephala iheringi (Platyhelminthes:Temnocephalida) based on specimens from Pomacea canaliculata (Mollusca: Ampullariidae) of the state of Rio Grande do Sul, Brazil: the possible type host and type locality. Rev. Bras. Zool. 27:245-257.

SEIXAS, S.A., AMATO, J.F.R. \& AMATO, S.B. 2010b. First report of Temnocephala haswelli (Platyhelminthes: Temnocephalida) in Pomacea canaliculata (Mollusca: Ampullariidae) from Brazil: description update based on specimens from the state of Rio Grande do Sul, Brazil. Zoologia 27:455-464. http://dx.doi.org/10.1590/S1984-46702010000300020

SEIXAS, S.A., AMATO, J.F.R. \& AMATO, S.B. 2010c. First report of Temnocephala rochensis (Platyhelminthes: Temnocephalida) from Pomacea canaliculata (Mollusca: Ampullariidae) outside Uruguay description update based on specimens from the state of Rio Grande do Sul, Brazil. Zoologia 27:820-828. http://dx.doi.org/10.1590/S198446702010000500019

SLUYS, R., HAUSER, J. \& WIRTH, Q.J. 1997. Deviation from the Groundplan: a unique new species of freshwater planarian from South Brazil (Platyhelminthes, Tricladida, Paludicola). J. Zool. 241:593-601. http://dx.doi.org/10.1111/j.1469-7998.1997.tb04851.x

TOURENQ, C., BENNETTS, R.E., KOWALSKI, H., VIALET, E., LUCCHESI, J.L., KAYSER, Y. \& ISENMANN, P. 2001. Are ricefields a good alternative to natural marshes for waterbird communities in the Camargue, southern France? Biol. Conserv. 100(3):335-343. http://dx.doi. org/10.1016/S0006-3207(01)00037-4

YOUNG, J.O. 2001. Keys to the freshwater microturbellarians of Britain and Ireland with notes on their ecology. Freshwater biological association scientific publication, Ambleside. 